\title{
ПРИМЕНЕНИЕ ПРИНЦИПА МАКСИМУМА ДЛЯ ОПТИМИЗАЦИИ ЦЕПЕЙ
}

\author{
А. М. ЗЕМЛЯК \\ ${ }^{1}$ Нацииональный технический университет Украины \\ «Киевский политехнический институт им. Игоря Сикорского», \\ Украина, Киев, 03056, пр-т Победь 37 \\ ${ }^{2}$ Автономный университет, \\ Мексика, Пуэбла,
}

\begin{abstract}
Аннотация. Решение проблемы сокращения затрат машинного времени при оптимизации электронных цепей позволяет улучшить качество проектирования. Обобщенная методология оптимизации цепей, развитая ранее на основе теории оптимального управления, позволила определить множество различных стратегий оптимизации. Определение функции Ляпунова процесса оптимизации и ее анализ для различных стратегий позволили сравнить эти стратегии с точки зрения вычислительных затрат и выбирать наилучшие из них. В то же время, наиболее обоснованным подходом для поиска оптимальной стратегии проектирования, в данной формулировке, является принцип максимума Понтрягина. Однако, использование этого принципа для решения нелинейных задач сопряжено со значительными трудностями. В настоящей работе получено решение задачи оптимизации электронной цепи за минимально возможное время процессора на основе принципа максимума Понтрягина в общем случае $N$ переменных. Показано, что изученный ранее эффект ускорения процесса оптимизации совпадает с решением, полученным на основе принципа максимума. Этот факт является теоретическим обоснованием эффекта ускорения. С другой стороны принцип максимума может стать основой для разработки алгоритма оптимизации электронных цепей с минимальными затратами процессорного времени.
\end{abstract}

Ключевые слова: аналоговые цепи; обобщенная методология оптимизации; принцип максимума Понтрягина

\section{ВВЕДЕНИЕ}

Возможность сокращения процессорного времени, необходимого для достижения точки минимума целевой функции процесса оптимизации, может быть реализована путем сокращения времени анализа системы и улучшения оптимизационных процедур. Благодаря разреженной структуре матрицы проводимости электронной схемы многие идеи для работы с разреженными матрицами успешно реализованы [1]. Иной путь уменьшения числа операций для анализа электронных схем связан с идеей декомпозиции [2].
Различные методы оптимизации аналоговой схемы могут быть классифицированы на две основные группы: детерминированные алгоритмы оптимизации и стохастические алгоритмы поиска. Некоторые недостатки классических детерминированных алгоритмов оптимизации состоят в требовании хорошего начального приближения в пространстве параметров, неудовлетворительного локального минимума, который может быть достигнут, и очень часто в требовании непрерывности и дифференцируемости целевой функции.

Чтобы преодолеть эти проблемы, применяются некоторые специальные методы. На- 


\section{БИБЛИОГРАФИЧЕСКИЙ СПИСОК}

1. George, A. On block elimination for sparse linear systems. SIAM J. Numer. Anal., Vol. 11, No. 3, P. 585-603, 1984. DOI: $10.1137 / 0711050$.

2. Ruehli, A.; Sangiovanni-Vincentelli, A.; Rabbat, G. Time analysis of large-scale circuits containing one-way macromodels. IEEE Trans. Circuits Syst., Vol. 29, No. 3, P. 185-190, 1982. DOI: 10.1109/TCS.1982.1085127.

3. Stehr, G.; Pronath, M.; Schenkel, F.; Graeb, H.; Antreich, K. Initial sizing of analog integrated circuits by centering within topology-given implicit specifications. Proc. of Int. Conf. on Computer Aided Design, 9-13 Nov. 2003, San Jose, CA, USA. IEEE, 2003, P. 241-246. DOI: 10.1109/ICCAD.2003.159696.

4. Ojha, A. K.; Ota, R. R. Multi-objective geometric programming problems with cost coefficients as multiple parameters. Advanced Modelling and Optimization, Vol. 15, No. 3, P. 815-838, 2013. URL: https://camo.ici.ro/journal/vol15/v15c18.pdf.

5. Cervantes-Gonzalez, J. C.; Rayas-Sanchez, J. E.; Lopez, C. A.; Camacho-Perez, J. R.; Brito-Brito, Z.; Chavez-Hurtado, J. L. Space mapping optimization of handset antennas considering EM effects of mobile phone components and human body. Int. J. RF Microwave Computer-Aided Engineering, Vol. 26, No. 2, P. 121-128, 2016. DOI: $10.1002 /$ mmce.20945.

6. Ridzuan, M. R. M.; Hassan, E. E.; Abdullah, A. R.; Bahaman, N.; Kadir, A. F. A. A new meta heuristic evolutionary programming (NMEP) in optimizing economic energy dispatch. J. Telecommunication Electronic and Computer Engineering, Vol. 8, No. 2, P. 35-40, $2016 . \quad$ URL: http://journal.utem.edu.my/index.php/jtec/article/view/94 2.

7. Yengui, F.; Labrak, L.; Frantz, F.; Daviot, R.; Abouchi, N.; O'Connor, I. A hybrid GA-SQP algorithm for analog circuits sizing. Circuits and Systems, Vol. 3, No. 2, P. 146-152, 2012. DOI: 10.4236/cs.2012.32019. 
8. Cohen, M. W.; Aga, M.; Weinberg, T. Genetic algorithm software system for analog circuit design. Procedia CIRP, Vol. 36, P. 17-22, 2015. DOI: 10.1016/j.procir.2015.01.033.

9. Farago, P.; Csipkes, G.; Csipkes, D.; Farago, C.; Hintea, S. An FPAA approach to adaptive filter design with evolutionary software-driven reconfiguration. Electronika ir Electrotechnika, Vol. 20, No. 5, P. 89-96, 2014. DOI: $10.5755 / \mathrm{j} 01$. eee.20.5.5579.

10. Srivastava, A.; Kachru, T.; Sylvester, D. Low-power-design space exploration considering process variation using robust optimization. IEEE Trans. Computer-Aided Design of Integrated Circuits and Syste$m s$, Vol. 26, No. 1, P. 67-79, 2007. DOI: 10.1109/TCAD.2006.882491.

11. Bosman, P. A. N. On gradients and hybrid evolutionary algorithms for real-valued multiobjective optimization. IEEE Trans. Evolutionary Computation, Vol. 16, No. 1, P. 51-69, 2012. DOI: 10.1109/TEVC.2010.2051445.

12. Fallahpour, M. B.; Hemmati, K. D.; Pourmohammad, A. Optimization of a LNA using genetic algorithm. Electrical and Electronic Engineering, Vol. 2, No. 2, P. 38-42, 2012.

13. Moradi, M. H.; Abedini, M. A combination of genetic algorithm and particle swarm optimization for optimal DG location and sizing in distribution systems. Int. J. Electrical Power \& Energy Systems, Vol. 34, No. 1, P. 66-74, 2012. DOI: 10.1016/j.ijepes.2011.08.023.

14. Engelbrecht, A. Particle swarm optimization. Proc. of 2014 Annual Conf. on Genetic and Evolutionary Computation, 2014, P. 381-406. DOI: $10.1145 / 2598394.2605342$.

15. Каширский, И.С.; Трохименко, Я.К. Обобщенная оптимизация электронных схем. К.: Техника, 1979. $192 \mathrm{c}$.

16. Rizzoli, V.; Costanzo, A.; Cecchetti, C. Numerical optimization of broadband nonlinear microwave circuits. IEEE MTT-S Int. Symp. Dig., 8-10 May 1990, Dallas, TX, USA. IEEE, 1990, Vol. 1, P. 335-338. DOI: $\underline{10.1109 / M W S Y M .1990 .99588}$.
17. Ochotta, E. S.; Rutenbar, R. A.; Carley, L. R. Synthesis of high-performance analog circuits in ASTRX/OBLX. IEEE Trans. Computer-Aided Design of Integrated Circuits and Systems, Vol. 15, No. 3, P. 273-294, 1996. DOI: 10.1109/43.489099.

18. Zemliak, A. Analog system design problem formulation on the basis of control theory. Proc. of 8th IEEE Int. Conf. on Electronics, Circuits and Systems, ICECS, 2-5 Sept. 2001, Malta. IEEE, 2001. DOI: 10.1109/ICECS.2001.957684.

19. Zemliak, A. Analog circuit optimization on basis of control theory approach. COMPEL: The International Journal for Computation and Mathematics in Electrical and Electronic Engineering, Vol. 33, No. 6, P. 2180-2204, 2014. DOI: 10.1108/COMPEL-10-2013-0324.

20. Zemliak, A.; Markina, T. Behavior of Lyapunov's function for different strategies of circuit optimisation. Int. J. Electronics, Vol. 102, No. 4, P. 619-634, 2015. DOI: 10.1080/00207217.2014.936046.

21. Понтрягин, Л.С.; Болтянский, В.Г.; Гамкрелидзе, Р.В.; Мищенко, Е.Ф. Математическая теория оптимальных процессов. М.: Наука, 1969. 384 с.

22. Rosen, J. B. Iterative solution of nonlinear optimal control problems. SIAM J. Control, Vol. 4, No. 1, P. 223-244, 1966. DOI: 10.1137/0304021.

23. Федоренко, Р. П. Приближенное решение задач оптимального управления. М.: Наука, 1978. 488 с.

24. Bourdin, L.; Trelat, E. Pontryagin maximum principle for finite dimensional nonlinear optimal control problems on time scales. SIAM J. Control Optim., Vol. 51, No. 5, P. 3781-3813, 2013. DOI: 10.1137/130912219.

25. Kamocki, R. Pontryagin maximum principle for fractional ordinary optimal control problems. Mathematical Methods in the Applied Sciences, Vol. 37, No. 11, P. 1668-1686, 2014. DOI: 10.1002/mma.2928.

26. Земляк, А. М. Применение принципа максимума Понтрягина для задачи оптимизации цепей // Tруды VII Всероссийской научно-технической конференции «Проблемы разработки перспективных микро- и наноэлектронных систем 2016», Т. 1, С. 94-100, 2016. 